- ART is a successful method that uses glass ionomer cement to control decay.

- Although ART has good success rates, it is not known how ionometric material can affect dentine structure.

- Microhardness studies may help investigations of tooth structure alterations like remineralisation and demineralisation.

\title{
Microhardness of dentine underlying ART restorations in primary molars: an in vivo pilot study
}

\author{
B. M. Santiago, ${ }^{1}$ D. A. Ventin, ${ }^{2}$ L. G. Primo ${ }^{3}$ and R. Barcelos ${ }^{4}$
}

Objective To evaluate the microhardness of dentine underlying glass ionomer restorations made with Atraumatic Restorative Treatment. Design An experimental single-centre study. Setting University Department, Brazil, 2001.

Materials and Methods Sixteen children were selected showing primary molars containing carious cavities $(n=29)$, which were restored using Fugi IX. Subsequently, some teeth were extracted respecting Nolla stage 7 or 8 of the permanent successor, and categorised according to the post-restoration time in the mouth: $\mathrm{G} 1$ (baseline, immediate extraction), G2 (30 days), G3 (90 days) and G4 (180 days). Four teeth were excluded because they were not in time of normal exfoliation. The 25 extracted teeth were resin embedded, sectioned mesiodistally and prepared metallographically. Knoop microhardness analysis (Micromet 2003; $10 \mathrm{~g}$ for $15 \mathrm{sec}$.) produced identations in three areas of dentine: Zone 1 (just below the restoration), Zone 3 (as close as possible to the pulp) and Zone 2 (intermediate region between 1 and 3). Nonparametric statistical tests were done - at a significance level of 5\%. Results Four teeth were excluded. No significant difference was noted among the zones, inside the groups. Zones 1 and 2 showed a considerable increase of microhardness according to the time, except for Group 3 ( $p>0.05$ ).

Conclusion Although microhardness had increased, it was not sufficiently equal to the microhardness of the healthy dentine, regardless of the depth evaluated.

\section{INTRODUCTION}

Atraumatic Restorative Treatment (ART) is a method used for removing carious tissue by excavating with hand instruments and immediately filling the cavity with glass ionomer cement (GIC). ${ }^{1-3}$

\footnotetext{
${ }^{1 *}$ Paediatric Dentistry Master, Department of Pediatric Dentistry and Orthodontics, Federal University of Rio de Janeiro, Rio de Janeiro, Brazil; ${ }^{2}$ Paediatric Dentistry Specialist, Department of Pediatric Dentistry and Orthodontics, Federal University of Rio de Janeiro, Rio de Janeiro, Brazil: ${ }^{3}$ Adjunctive professor, Department of Pediatric Dentistry and Orthodontics, Federal University of Rio de Janeiro, Rio de Janeiro, Brazil; ${ }^{4}$ Paediatric Dentistry Master, Department of Pediatric Dentistry and Orthodontics, Federal University of Rio de Janeiro, Rio de Janeiro, Brazil.

*Corresponding author: Bianca Marques Santiago - Rua Baltazar Lisboa, 25/303, Tijuca, Rio de Janeiro/RJ, Brazil. Tel: 55(21)2569-3875. Fax: 55(21)2254-6808.

Email:biasantiago@openlink.com.br
}

\section{Refereed paper}

Received 03.02.03; Accepted 08.12.03

doi: 10.1038/sj.bdj.4812525

(๑) British Dental Journal 2005; 199: 103-106
The literature has indicated clinical success for ART, in primary teeth, varying from 80-90\% for restorations of one surface and from $55-75 \%$ for restorations involving more than one surface, after a one year follow-up. In permanent teeth, the rate of success is $90 \%$ for simple restorations followed for 2-3 years, although there are few studies reporting restorations with more than one surface. ${ }^{4}$

The advantage of using GIC lies in some of its properties, like liberation of fluoride, simplicity and a good cost-effectiveness ratio. ${ }^{5}$ The fluoride released by these restorative materials will be incorporated by the enamel and dentine, increasing its resistance to demineralisation from bacterial attack. ${ }^{6-9}$ There is no formation of an initial lesion or recurrence of caries in cavity walls restored with GIC. ${ }^{10}$ The carious process ceases and remineralisation of the affected dentine occurs. However, more studies are needed to confirm the effectiveness of the remineralisation and the extent to which it occurs. It is also important to determine the speed of that remineralisation. ${ }^{10}$

There are few studies on the influence of ionomeric material on tooth structure, especially carious dentine, left under the restorations as recommended in ART. Accordingly, the purpose of this study was to investigate whether microhardness increases, during time, in the dentine underlying the glass ionomer restorations placed using the ART method.

\section{MATERIALS AND METHODS}

Patients aged between nine and 11 years, who were on a waiting list for treatment in a Primary Care Clinic of a public university in Rio de Janeiro, were treated. The patients were examined and those that had primary molars with dentinal caries, with an opening greater than $3 \mathrm{~mm}$, covering one surface, without painful symptoms or clinical signs of pulp involvement, were selected. Radiographs were also taken using the parallelism technique, ${ }^{11}$ to determine the development stage of the permanent successors, which should be close to Nolla stages 7 and $8 .{ }^{12}$

This research project was approved by the Ethics in Human Research Committee of the University and treatment was only offered to patients whose guardians had signed a statement of consent.

The 29 teeth (16 patients) chosen had the carious tissue partially removed under isolation with cotton wool rolls ${ }^{3,13}$ and received ART restorations with Fuji IX handmixed cement (GC Corporation - Japan - 0107041), mixed in the proportion of one part of dust for 
one part of liquid, according to the manufacturer's instructions. After filling the cavity and applying varnish, before the final setting of the material, the patient was asked to occlude. Then, the surplus material was removed with excavators and varnish was applied again.

The teeth were then divided into four groups according to the time of permanence in the buccal cavity (Fig. 1): Group 1, teeth extracted immediately after filling, in the first appointment $(n=6)$; Group 2, teeth extracted or exfoliated one month after filling $(n=5)$; Group 3, teeth extracted or exfoliated three months after the first appointment $(n=5)$; and Group 4, teeth exfoliated or extracted after six months after filling, in the final check-up appointment $(\mathrm{n}=9)$. As there would be a long time interval between the check-up appointments (30, 90 and 180 days), bottles of formol saline solution (10\%) were given to the patient's guardian, who was requested to write the date and store the tooth, in the case of spontaneous exfoliation. Four teeth were excluded because they were not in time of normal exfoliation during the study period.

After exfoliation or extraction, 25 teeth were embedded in epoxy resin (XR 1303/XGY 1109-Araltec ${ }^{\circledR}$-Brazil) to avoid displacement of the restorations during sectioning. Teeth were then sectioned with an Isomet (Low speed saw-Buhler ${ }^{\circledR}$-USA) perpendicular to the occlusal surface, mesiodistally. After that, two sections (vestibular (A), and lingual (B)) were obtained, exposing the middle portion of each tooth, consisting of healthy and carious dentine and also a part of the filling (Fig. 2). These surfaces were epoxy resin embedded, using a $1.5 \mathrm{~cm}$ diameter and $2 \mathrm{~cm}$ high plastic matrix, to be polished metallographically using No. 180, 400, 600 and 1000 file, under water refrigeration (Knuth Rotor - Struers device). Knoop hardness test was carried out, ${ }^{14}$ with a load of $10 \mathrm{~g}$ for 15 sec. (Micromet 2003 - Buehler Ltda - USA).

Three penetrations were made in each of the following zones: Zone 1 (next to the restoration), Zone 3 (as near as possible to the pulp) and Zone 2 (intermediate region between Zone 1 and Zone 3 ), so that the test could comprise the different areas of the dentine. Furthermore, that procedure was done twice on each surface of the samples, forming two vertical columns of indentations (Fig. 3). This meant that four average values of Knoop hardness were obtained for each zone, which later were transformed into a single value, through the arithmetic average.

The data were tabulated in the SPSS programme (version 10.0) and non parametric statistical tests were done (Friedman Test, Kruskal Wallis Test and Mann-Whitney Test with Bonferroni Correction). The level of significance was established at $5 \%$.

\section{RESULTS}

The results show that there is a variation of dentine hardness as the distance between the restoration and the identation area increases. However, it was not possible to find a significant statistical difference $(p<0.05)$ when the different zones were compared in each group by the Friedman Test.

As for the time interval, significant differences were found in Zones $1(p=0.044)$ and $2(p=0.033)$, when the Kruskall Wallis test was used (Table 1). However, the same table indicates that Zone 3 did not show a difference in the four groups ( $p=0.549$ ), a fact that was repeated in all the subsequent analyses.

The Mann-Whitney test was used to compare G1 with the others, in the different zones. With the division into different intervals of time, it was noted that the microhardness increased in the first 30 days $(\mathrm{G} 2 \times \mathrm{G} 1)$ in Zone $1(\mathrm{p}=0.030)$ and in Zone 2 $(\mathrm{p}=0.004)$. Also, a statiscally significant increase in hardness was found in the teeth that remained in situ for 180 days (G4 x G1) (finding $p=0.035$ for Zones 1 and 2). However, the same trend was not established when comparing G1 (immediate) with

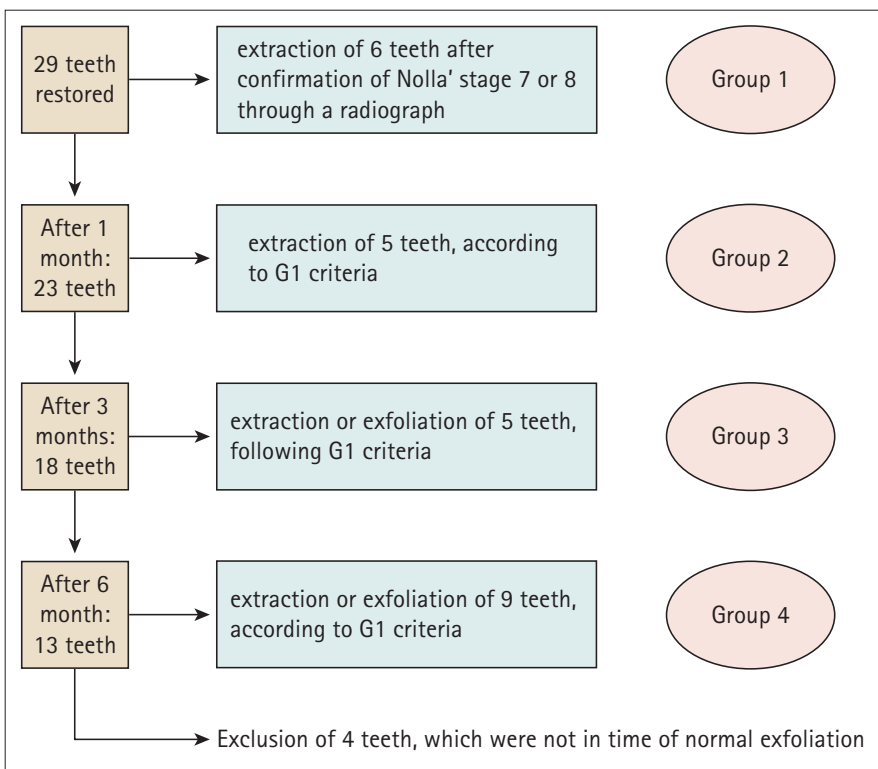

Fig. 1 Scheme of division of teeth studied in four groups, according to postrestoration time.

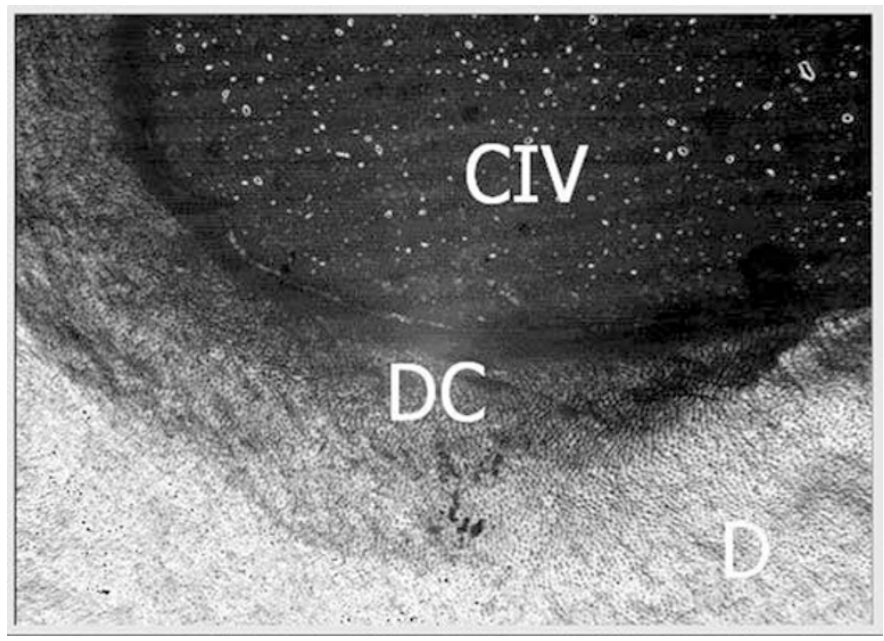

Fig. 2 View of one of the samples under an optical microscope (50x): (CIV) Glass ionomer cement; (DC) Carious Dentine; (D) Healthy Dentine.

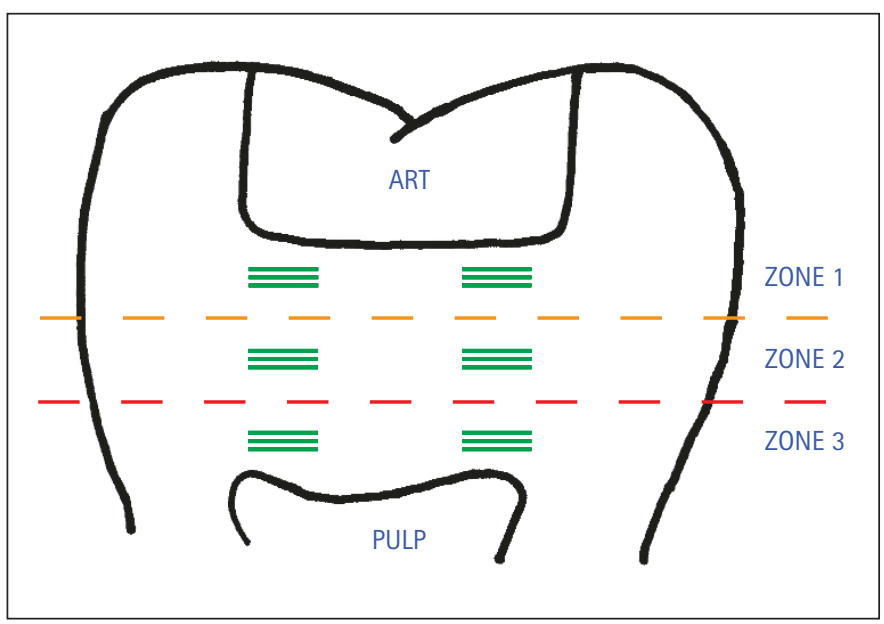

Fig. 3 Schematic drawing of the sites of identations in dentine, made during the microhardness test.

G3 (90 days) in Zones $1(p=0.931)$ and $2(p=0.429)$. Figure 4 illustrates the microhardness values obtained from each zone in the different time intervals. 


\begin{tabular}{|c|c|c|c|c|c|c|c|c|c|c|c|}
\hline \multirow{3}{*}{ Median } & \multicolumn{3}{|c|}{ Zone 1} & \multicolumn{4}{|c|}{ Zone 2} & \multicolumn{4}{|c|}{ Zone 3} \\
\hline & \multicolumn{3}{|c|}{ Interquartiles Ranges } & \multirow{2}{*}{ Median } & \multicolumn{3}{|c|}{ Interquartiles Ranges } & \multirow{2}{*}{ Median } & \multicolumn{3}{|c|}{ Interquartiles Ranges } \\
\hline & 25 & 50 & 75 & & 25 & 50 & 75 & & 25 & 50 & 75 \\
\hline 17,79 & 15,51 & 17,79 & 30,08 & 24,52 & 16,92 & 24,52 & 27,73 & 24,57 & 19,57 & 24,57 & 35,39 \\
\hline 36,41 & 32,93 & 36,41 & 50,26 & 50,19 & 32,88 & 50,19 & 57,29 & 35,55 & 31,15 & 35,55 & 40,85 \\
\hline 21,18 & 15,04 & 21,18 & 33,97 & 33,26 & 15,76 & 33,26 & 45,20 & 30,24 & 14,86 & 30,24 & 39,46 \\
\hline 42,07 & 32,42 & 42,07 & 49,34 & 32,29 & 26,96 & 32,29 & 43,59 & 34,82 & 19,74 & 34,82 & 42,05 \\
\hline \multicolumn{4}{|c|}{$p=0.444$} & \multicolumn{4}{|c|}{$p=0.033$} & \multicolumn{4}{|c|}{$p=0.549$} \\
\hline
\end{tabular}

Table 1 Microhardness values according to Time for Zones 1, 2 and 3 (KHN), Kruskal Wallis Test.

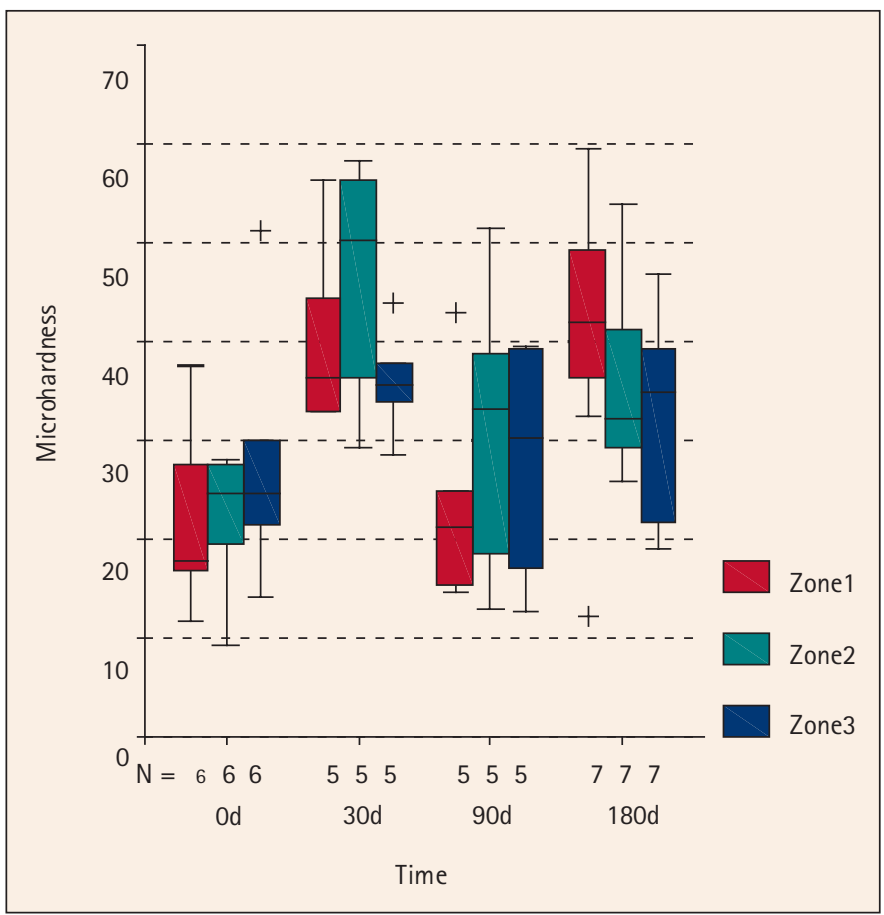

Fig. 4 Microhardness values obtained from the three zones in the different time intervals.

\section{DISCUSSION}

This pilot clinical study involves methodological difficulties inherent to any in vivo research, although it permits a more realistic appraisal of intraoral phenomena. One of the difficulties faced was to obtain the sample, due to the necessary characteristics for including volunteers in the research (carious primary molars, verging on exfoliation). At this point an ethical issue also arose, because the teeth could only be extracted at the time of normal exfoliation, which in some cases greatly increased the observation time, resulting in the exclusion of four elements.

Measuring microhardness makes it possible to evaluate the mineral content of the tissue, and to assess whether there was loss of mineral due to dissolution of the inorganic part, as happens during the carious process ${ }^{15}$, and also to quantify the gain in density through a process of ion incorporation (remineralisation). ${ }^{7}$

The load used to make the identations in that study was $10 \mathrm{~g}$, which is very small if compared with other experiments that used $50 \mathrm{~g}$ for testing enamel and/or dentine. ${ }^{7,16,17}$ However, the structure to be analysed was very delicate and it was not feasible to use larger loads as Craig, Gehring and Peyton ${ }^{18}$ suggested.

It was decided to use three zones for analysing the microhardness because dentine is not a homogenous tissue. ${ }^{18,19}$ The experimental model consisted of 18 indentations and attempted to minimise the interferences that could occur because of the preparation and testing of the samples. Other studies using that test also made a large number of identations. ${ }^{7,16,17}$

The microhardness values of the dentine found in this work differ from those reported in the literature. ${ }^{14,17}$ The figures obtained are below the range proposed by Craig ${ }^{14}$ and Meredith et al. ${ }^{17}$ which vary from 50 to $70 \mathrm{KHN}$, showing the different conditions under which this research was done, when it was sought to evaluate the carious dentine left under the ART. Nonetheless, the results are in accordance with the microhardness values of the carious dentine proposed by Craig, Gehring and Peyton, ${ }^{18}$ recorded at loads which varied from 19 to $34 \mathrm{KHN}$.

Furthermore, Gao, Smales and Yip ${ }^{20}$ reported that caries progresses in the dentine through three phenomena: demineralisation by the dissolution of the inorganic part, degeneration of the organic part and bacterial invasion. However, due to structural histological differences, two types of carious dentine can be recognised. Carious dentine that is more internal and deep, maintains the interlaced structure of the collagenous fibres and can be remineralised, resulting in increased hardness and calcium content, which is supplied by the pulp through odontoblastic processes. While in more external carious dentine, structural loss and odontoblastic processes occur. Therefore that layer is not capable of remineralisation. The slight increase of the calcium content is due to a mere diffusion of that ion and not to a physiological remineralisation process, with recovery of hardness. Thus, the suggestion is open that in some teeth the identation has been done in that layer of dentine which is not capable of recovering hardness, justifying the low values found.

A minor variation was found between the different dentine zones inside each group, although without a significant statistical difference. Hence, it was clear that although it is a heterogeneous tissue, the larger tubular structure next to the pulp did not result in a significant reduction of microhardness. A fact that was also noted by Craig and Peyton. ${ }^{16}$

Post-restoration time influenced zones 1 and 2, although zone 3 did not show any statistically significant difference $(p=0.549)$ among the four groups, suggesting that the dentine closest to the pulp and farther from the restoration was not affected as to hardness, either by incorporation of elements released by the restorative material or by a process of physiological reparation.

When comparing G1 (baseline) with the others, it was not possible to verify a significant increase of microhardness 
compared to the G3 teeth (90 days). It is necessary to consider two situations to explain that finding. The first relates to the fact that the G3 is composed of teeth with more extensive and deeper restorations. It is suggested that teeth that have an extensive carious process undergo greater mineral loss, in addition to the possibility of the identation having been done in an area of carious dentine that could not be remineralised. ${ }^{20}$ One of the methodological limitations of this pilot study fits that situation, in which the minimum opening size of the cavities was standardised, but not the maximum depth.

The second possible explanation is more complex and involves a series of factors studied separately. At first, the microhardness increases in 30 days, which could be due to the initial release of fluoride by the ionomeric cements being greater in the beginning. ${ }^{21}$ However, according to Wandera, ${ }^{9}$ fluoride does not cause remineralisation, it just forms a free/loose layer. It is suggested, therefore, that with a continuous process of de-remineralisation, that loose layer could be lost before the actual mineral content of the tooth.

In an in situ study, ten Cate and van Duinen ${ }^{6}$ noted the occurrence of hypermineralisation in the tissue underlying GIC restorations. They concluded that the increase of resistance to demineralisation could be attributed to a mechanical barrier formed by the deposition of mineral on the dentinal pores, limiting the diffusion of the acids originating in the plaque. Contrary to that situation, this experiment suggested that some increase of the inorganic content occurs, because the microhardness increased.

Samuel and Rubinstein ${ }^{7}$ found, through microhardness analysis, that Vitremer (resin modified glass ionomer cement) caused some phenomenon on the adjacent enamel, because it did not show a difference for the control group, without restoration, after they had been submitted to alternating demineralisation-remineralisation cycles in vitro. However, no follow-up was carried out, nor was the dentine evaluated.

The results of this pilot study suggest that the increase of microhardness of the dentine adjacent to the GIC should not be attributed only to the release of fluoride, but also to the suspension of the carious process through an adhesive restoration, which prevents the substrate being reached by cariogenic microorganisms. Six, Lasfargues and Goldberg ${ }^{22}$ suggested the same, when they noted, through a histological study of rat teeth, that GIC neither inhibits nor stimulates the formation of reparative dentine when compared to the self-reparation process (unrestored cavities).

According to this reasoning, it is possible to explain the increased microhardness after 180 days, because once the source of nutrition for the cariogenic bacteria is eliminated, the microorganisms will not survive, putting an end to the carious process. ${ }^{23}$ An adhesive and sealed composite restoration, over a lesion with overt cavitation, can establish a physical barrier against nutrients originating from the buccal cavity. Without them, the cariogenic microorganisms under the restoration will not be able to go through their metabolism, leading to suspension of the caries. Once sealed, those lesions will probably stabilise and not progress as was noted by Mertz-Fairhurst et al. ${ }^{23}$ in their 10-year clinical follow-up. Van Amerongen ${ }^{24}$ had already described that phenomenon and emphasised that this situation can be expected with the use of any material capable of hermetically sealing the cavity.

\section{CONCLUSION}

According to this clinical pilot study, it was possible to conclude that the microhardness of the dentine under ART restorations increases over time, although that increase is not sufficient for the microhardness to reach a value similar to that of healthy dentine, regardless of the depth observed. Moreover, additional studies are necessary to verify the influence of the fluoride release, the dentine microhardness increase and to base the influence of the time factor on the dentine reparation process.

The authors are grateful to the Mechanical Testing Laboratory of the Military Engineering Institute of Rio de Janeiro, RJ, Brazil, for its cooperation through specialist Leonardo F. Cruz.

1. Ewoldsen N, Covey D and Lavin M. The physical and adhesive properties of dental cements used for atraumatic restorative treatment. SDC Special Care in Dentistry 1997; 17: 19-24.

2. Phantumvanit $P$, Song paisan $Y$, Frencken J E, Pilot T. Atraumatic restorative treatment technique - Evaluation after one year. J Dent Res 1995; 73: 1006, Abst No. 30 .

3. Phantumvanit $P$, Songpaisan $Y$, Frencken J E, Pilot T. Atraumatic restorative treatment technique - Evaluation after two years. J Dent Res 1995; 73: 1014, Abst. No. 24.

4. Smales R J, Yip H K. The atraumatic restorative treatment (ART) approach for the management of dental caries. Quintessence Int 2002; 33: 427-432.

5. Phantumvanit $P$, Songpaisan $Y$, Frencken J E, Pilot T. Atraumatic restorative treatment (ART): Evaluation after two years. p 53. WCPD'95 Official Program, 1995.

6. ten Cate J M, van Duinen R N B. Hypermineralisation of dentinal lesions adjacent to glass-ionomer cement restorations. J Dent Res 1995; 74: 1266-1271.

7. Samuel S M W, Rubinstein C. Microhardness of enamel restored with fluoride and non-fluoride releasing dental materials. Braz Dent J 2001; 12:35-38.

8. Smales R J, Gao W. In vitro caries inhibition at the enamel margins of glass ionomer restoratives developed for the ART approach. J Dent 2000; 28: 249-256.

9. Wandera A. In vitro enamel effects of a resin-modified glass ionomer: fluoride uptake and resistance to demineralisation. Pediatr Dent 1998; 20: 411-417.

10. Mount G J. Some physical and biological properties of glass ionomer cement. Int Dent J 1995; 45: 135-140.

11. Lyre W R, Johnson 0 N. Essentials of dental radiography for dental assistants and hygienists. pp 231-250. Norwalk: Appleton \&t Lange, 1995.

12. Nolla C M. The development of permanent teeth. J Dent Child 1960; 4: 254-266.

13. Pilot $T$, Frencken J, Phantumvanit $P$, Song paisan $Y$. A prevention oriented oral care system for rural and underprivileged population. Adv Dent Res 1995; 9: 155.

14. Craig R G. Restorative dental materials. pp 54-105. St. Louis: Mosby, 1993.

15. Kodaka T, Debari K, Yamada M, Kuroiwa M. Correlation between microhardness and mineral content in so und human enamel. Caries Res 1992: 26: 139-141.

16. Craig R G, Peyton FA. The microhardness of enamel and dentin. J Dent Res 1958; 37: $661-668$.

17. Meredith N, Sherriff M, Setchell D J, Swanson S A V. Measurement of the microhardness and young's modulus of human enamel and dentine using an indentation technique. Arch Oral Biol 1996; 41: 539-545.

18. Craig R G, Gehring P E, Peyton FA. Relation of structure to the microhardness of human dentin. J Dent Res 1959; 38: 624-630.

19. Smith D C, Williams D F. Biocompatibility of dental materials. Volume I. pp 75-95 Florida: CRC Press, 1982

20. Gao W, Smales R J, Yip H K. Demineralisation and remineralisation of dentine caries and the role of glass-ionomer cements. Int Dent J 2000; 50: 51-56.

21. Carvalho A S, Cury J A. Fluoride release from some dental materials in different solutions. Oper Dent 1999; 24: 14-19.

22. Six N, Lasfargues J J, Goldberg M. In vivo study of the pulp reaction to Fugi IX, a glass ionomer cement. J Dent 2000; 28: 413-422.

23. Metz-Fairhurst E J, Curtis J W, Ergle J W, Rueggeberg FA, Adair S M. Ultraconservative and cariostatic sealed restorations: Results at year 10. J Am Dent Assoc 1998; 129: 55-66.

24. van Amerongen W E. Dental caries under glass ionomer restorations. J Public Health Dent 1996; 56: 150-154 